\title{
VARIABILIDADE DOS PARÂMETROS DA EQUAÇÃO QUE RELACIONA A CONDUTIVIDADE HIDRÁULICA COM A UMIDADE DO SOLO NO MÉTODO DO PERFIL INSTANTÂNEO ${ }^{(1)}$
}

\author{
Q. deJ ONG van LIER ${ }^{(2)} \&$ P. L. LIBARDI ${ }^{(3)}$
}

\begin{abstract}
RESUMO
A condutividade hidráulica é uma das mais importantes propriedades do solo para estudos que envolvem a infiltração da água, o movimento da água dentro do perfil, bem como para as raízes das plantas e para a drenagem interna. O conhecimento da função condutividade hidráulica versus umidade $(K(\theta))$ é essencial para esses estudos; no entanto, a informação disponível sobre a variabilidade dos parâmetros empíricos dessa função é escassa. Com o objetivo de conhecer a variabilidade desses parâmetros, realizaram-se, neste trabalho, em doze locais do mesmo solo, observações da condutividade hidráulica em função da umidade do solo pelo método do perfil instantâneo, dentro de uma área de $1.000 \mathrm{~m}^{2}$. A equação utilizada para $K(\theta)$ foi $K=K_{0} \cdot \exp \left[\gamma\left(\theta-\theta_{0}\right)\right]$, em que $K_{0}$ e $\theta_{0}$ são os valores de $K$ e $\theta$, respectivamente, para o tempo zero de redistribuição da água. As medições de umidade e potencial mátrico foram feitas ao longo de 50 dias, aproximadamente, em nove profundidades entre 0,2 e 1,0 m. Os resultados mostraram que coeficientes de variação em torno de dois a três foram obtidos para os valores de $K_{0}$ na maioria das profundidades e que os valores de $\gamma$ apresentaram coeficientes de variação menores, em torno de 0,2 a 0,5, maiores na superfície e menores em profundidade. Concluiu-se que os valores de umidade versus tempo ajustaram-se muito bem a uma equação potencial e os de potencial total versus profundidade muito bem a uma equação polinomial de segundo grau. Os resultados indicaram que a relação de $K$ versus $\theta$, determinada pelo método do perfil instantâneo em uma área de alguns metros quadrados, não foi representativa de área muito maior.
\end{abstract}

Termos de indexação: Equação de Richards, tensiômetros, sonda de nêutrons.

\footnotetext{
(1) Recebido para publicação em janeiro de 1999 e aprovado em junho de 1999.

(2) Professor Doutor do Departamento de Ciências Exatas, Escola Superior de Agricultura Luiz de Queiroz - ESALQ/USP. Caixa Postal 9, CEP 13418-900 Piracicaba (SP). Bolsista do CNPq.

(3) Professor Titular do Departamento de Ciências Exatas, ESALQ/USP. Bolsista do CNPq.
} 


\title{
SUMMARY: PARAMETER VARIABILITY OF THE EQUATION RELATING HYDRAULIC CONDUCTIVITY TO SOIL WATER CONTENT USING THE INSTANTANEOUS PROFILE METHOD
}

\begin{abstract}
Hydraulic conductivity is one of the most important soil properties for studies dealing with infiltration, water movement within the soil profile and to plant roots and internal drainage. Theavailability of the function hydraul ic conductivity $x$ soil water content $(K(\theta))$ is essential to these studies. However, little is known about the variability of the empirical parameters of this function. Aiming to contribute with information on the variability of these parameters, a study is described in which observations of hydraul ic conductivity as a function of soil water content by the instantaneous profile method were made in twelve locations within an area of $1000 \mathrm{~m}^{2}$. The equation used for $\mathrm{K}(\theta)$ was $\mathrm{K}=\mathrm{K}_{0} \cdot \exp \left[\mathcal{\gamma}\left(\theta-\theta_{0}\right)\right]$, in which $\mathrm{K}_{0}$ and $\theta_{0}$ are $\mathrm{K}$ and $\theta$ values, respectively, for zero time of water redistribution. The measurements of soil water content and matric potential were performed for about 50 days in nine depths between 0.2 and $1.0 \mathrm{~m}$. Results show coefficients of variation of about two to threefor the majority of depths for the $K_{0}$ values, whileval ues for $\gamma$ showed lower variation coefficients, between 0.2 and 0.5 , thehigher occurring cl oseto thesurfaceand thelower ones at greater depths. It is concluded that soil water content data fit very well to time by a potential equation, while total water potential fits very well to depth by a second order polynomial equation. Results suggest that a relationship between $\mathrm{K}$ and $\theta$, determined by the instantaneous profile method in an area of a few square meters, is not representantive for a much larger area than that whereit was determined.
\end{abstract}

Index terms: Richards' equation, tensi ometers, neutron probe

\section{INTRODUÇÃO}

Entre as propriedades físicas do solo, a condutividadehidráulica éuma das mais importantes quandose estudam fenômenos que, deal guma forma, estão ligados ao movimento da água no solo. Dessa maneira, no contexto da agronomia, a condutividade hidráulica tem especial importância em estudos que envolvem a infiltração da água no solo (Kunze \& Nielsen, 1983; Swartzendruber, 1987; Wolfe et al, 1988), o movimento da água dentro do perfil de solo e para as raízes das plantas (Gardner \& Ehlig, 1962, Hainsworth \& Aylmore, 1986, 1989; J ong van Lier \& Libardi, 1997) e a drenagem interna da água no solo (Freire, 1979; Libardi \& Saad, 1994). Com exceção de algumas situações específicas, o solo agrícola revela umidade inferior à saturação e, pela existência de uma função crescente entre condutividade eumidade, oval or da condutividadehidráulica sob a condição saturada é sempre superior ao das condições não saturadas, para o mesmo solo. Conhecer a função condutividade hidráulica versus umidade ou, simplesmente, função $K(\theta)$, torna-se, portanto, essencial para esses estudos.

Diversos trabalhos abordam a variabilidade espacial das propriedades hídricas do solo, como os de Warrick \& Nielsen (1980), Vieira et al. (1981), Bouma et al. (1989), Moraes \& Libardi (1993) e Logsdon \& J aynes (1996). A síntesedesses trabalhos leva a concluir que as propriedades hídricas do solo, como a condutividade hidráulica, a capacidade de infiltração e a própria umidade, têm variabilidade espacial muitoalta.

Alguns trabalhos foram desenvolvidos determinando a função $K(\theta)$ e a sua variabilidade com utilização de um permeâmetro de Guel ph (Reynol ds \& Zebchuk, 1996; Russo et al., 1997). Por ele, determina-sea função $K(\theta)$ para o solo bem próximo à saturação.

Para determinar a função $K(\theta)$ da saturação até umidades menores, utiliza-se o método de perfil instantâneo, constante da literatura da década de 60 (Watson, 1966) e aperfeiçoado e simplificado por Hillel et al. (1972) e Libardi et al. (1980). A vantagem do método está no fato de basear-se em medições diretas no campo. A desvantagem está no investimento alto de tempo e mão-de-obra, especial mente em sol os com camadas adensadas ou compactadas, onde o processo de drenagem é muito lento . É por essa razão que são muito poucos os relatos sobre determinações de $K(\theta)$ pel o método de perfil instantâneo com número maior de repetições. A informação disponível sobre a variabilidade e confiabilidade detais medições é, portanto, escassa, concordando com a afirmação de Govindaraju et al. (1992) de que os métodos baseados na equação de Richards, como o do perfil instantâneo, embora teoricamentecorretos, acarretam grandes problemas em sua aplicação, em virtude da exigência de dados de entrada difíceis de ser obtidos com precisão. 
É comum encontrar estudos sobre balanço hídrico que se baseiam em apenas uma medida da condutividade hidráulica, considerada válida para uma área experimental inteira (Libardi \& Saad, 1994). Conformealegaram Webster \& Oliver (1992), a elaboração de um semivariograma de propriedade do solo com menos de 50 dados observados é de reduzido valor, sendo necessário um mínimo de aproximadamente 200 para uma análise confiável. O número 200, estimado por esses autores, está sujeito a correções, considerando a variabilidade da medida em questão. A condutividade hidráulica do solo está, reconhecidamente, entre as propriedades físi cas do sol o com a mais alta variabilidade. Warrick \& Nielsen (1980) apresentaram dados mostrando coeficiente de variação da ordem de $100 \%$, para a condutividade hidráulica do solo saturado, e da ordem de $400 \%$, para a condutividade hidráulica do sol o a uma umi dade $40 \%$ inferior à saturação. Sendo assim, 200 observações provavel menteainda seriam um número pequeno para análise desse tipo.

Considerando o grande e necessário número de observações, no presente estudo, não se pôde objetivar uma análise geoestatística. Porém, pretendeu-se conhecer a variabilidade de medições da relação entre condutividade hidráulica e umi dade do solo pelo método do perfil instantâneo por meio da realização de um número relativamente grande de observações desse tipo dentro de uma área pequena.

\section{MATE RIAL E MÉTODOS}

\section{Fundamentos teóricos}

No método do perfil instantâneo, procura-se uma solução para a equação de Richards:

$$
\frac{\partial \theta}{\partial t}=\frac{\partial}{\partial z}\left(K(\theta) \frac{\partial \psi_{t}}{\partial z}\right)
$$

em que $\theta\left(m^{3} m^{-3}\right)$ é a umi dade vol umétrica, $t(h)$ éo tempo, $\mathrm{K}\left(\mathrm{m}^{2} \mathrm{~h}^{-1} \mathrm{kPa}^{-1}\right)$ éa condutividadehidráulica, $\psi_{\mathrm{t}}(\mathrm{kPa})$ é o potencial total da água e $\mathrm{z}(\mathrm{m})$ é a coordenada vertical de posição. I ntegrando a equação de Richards com relação à profundidade $z$, entre os limites $z=0$ e $z=z$, com as seguintes condições de contorno:

$$
\begin{aligned}
& z=0 ; t>0 ; q=0 \\
& z=z ; t>0 ; q=q z
\end{aligned}
$$

obtém-se:

$$
\int_{0}^{\mathrm{z}} \frac{\partial \theta}{\partial \mathrm{t}} \mathrm{dz}=\left.\mathrm{K}(\theta) \frac{\partial \psi_{\mathrm{t}}}{\partial \mathrm{z}}\right|_{\mathrm{z}}=\mathrm{q}_{\mathrm{z}}
$$

ou, rearranjando

$$
\left.\mathrm{K}(\theta)\right|_{\mathrm{z}}=\frac{\int \frac{\mathrm{z} \theta}{\partial \mathrm{t}} \mathrm{dz}}{\left.\frac{\partial \psi_{\mathrm{t}}}{\partial \mathrm{z}}\right|_{\mathrm{z}}}=\frac{\frac{\partial \mathrm{h}_{\mathrm{z}}}{\partial \mathrm{t}}}{\left.\frac{\partial \psi_{\mathrm{t}}}{\partial \mathrm{z}}\right|_{\mathrm{z}}}
$$

em que $h_{z}\left(m^{3} m^{-2}\right)$ é a armazenagem de água no solo entrea superfície $(z=0)$ ea profundidade $z$. Por meio de medidas simultâneas de umidade e de potencial total ao longo do perfil de solo durante a redistribuição da água, determinam-se a variação de armazenagem $h_{z}$ com o tempo t e o gradiente de $\psi_{\mathrm{t}}$ ao longo dez que aparecem na equação 3 e, então, o valor de $\mathrm{K}(\theta)$.

Normal mente, os valores de $K(\theta)$ apresentam uma relação exponencial coma umidade, de maneira que a função $K(\theta)$ pode ser expressa pela equação

$$
K(\theta)=K_{0} e^{\gamma\left(\theta-\theta_{0}\right)}
$$

em que $\gamma$ é uma constante adimensional e $K_{0}$ $\left(\mathrm{m}^{2} \mathrm{~h}^{-1} \mathrm{kPa}-1\right)$ e $\theta_{0}\left(\mathrm{~m}^{3} \mathrm{~m}^{-3}\right)$ são valores de $\mathrm{K}$ e $\theta$ no tempo zero de redistribuição.

\section{Métodos de campo}

O trabal ho experimental de campo foi realizado durante os meses de junho e julho de 1995, numa área do campus "Luiz de Queiroz" em Piracicaba (SP). Constou de 12 pontos de observação, em uma grade de $3 \times 4$ pontos numa área de $1.000 \mathrm{~m}^{2}$ (Figura 1). N essa figura, verifica-se quea distância entre os locais de observação nos pontos extremos da área (entre ponto 1 e 12, ou 4 e9) é de pouco mais de $50 \mathrm{~m}$.

Próximo ao local, foi aberta uma trincheira para descrição pedológica do solo e para a retirada de amostras indeformadas para a determinação da densidade do sol o nos diferentes horizontes. O solo, que apresenta uma seqüência de horizontes $A_{p}-B_{t}-B_{w}$, com densidade de quase $1.600 \mathrm{~kg} \mathrm{~m}^{-3}$ no $A_{p}$ e entre 1.200 e $1.400 \mathrm{~kg} \mathrm{~m}^{-3}$ nas camadas mais profundas (Quadro 1), foi classificado como Terra Roxa Estruturada latossólica. O dedive no local é inferior a 0,01 $\mathrm{m} \mathrm{m}^{-1}$.

Em cada ponto foram instalados nove tensiômetros para a medição do potencial mátrico e um tubo de acesso de alumínio para sonda de nêutrons para a determinação da umidade. Os tensiômetros, com manômetro de mercúrio, foram instalados com as suas cápsulas porosas a cada 0,1 m de profundidade entre 0,2 e 1,0 m.

A sonda de nêutrons (503 Hydroprobe da CPN Corporation) era munida com uma fonte de ${ }^{241} \mathrm{Am} / \mathrm{Be}$ 
de intensidade radioativa de 1,85 GBq. O tempo de cada contagem foi de $30 \mathrm{~s}$ e, em cada profundidade, foram feitas três contagens por observação, obtendose valores típicos da ordem de grandeza de 2,5.104. A contagem da sonda de nêutrons foi expressa em

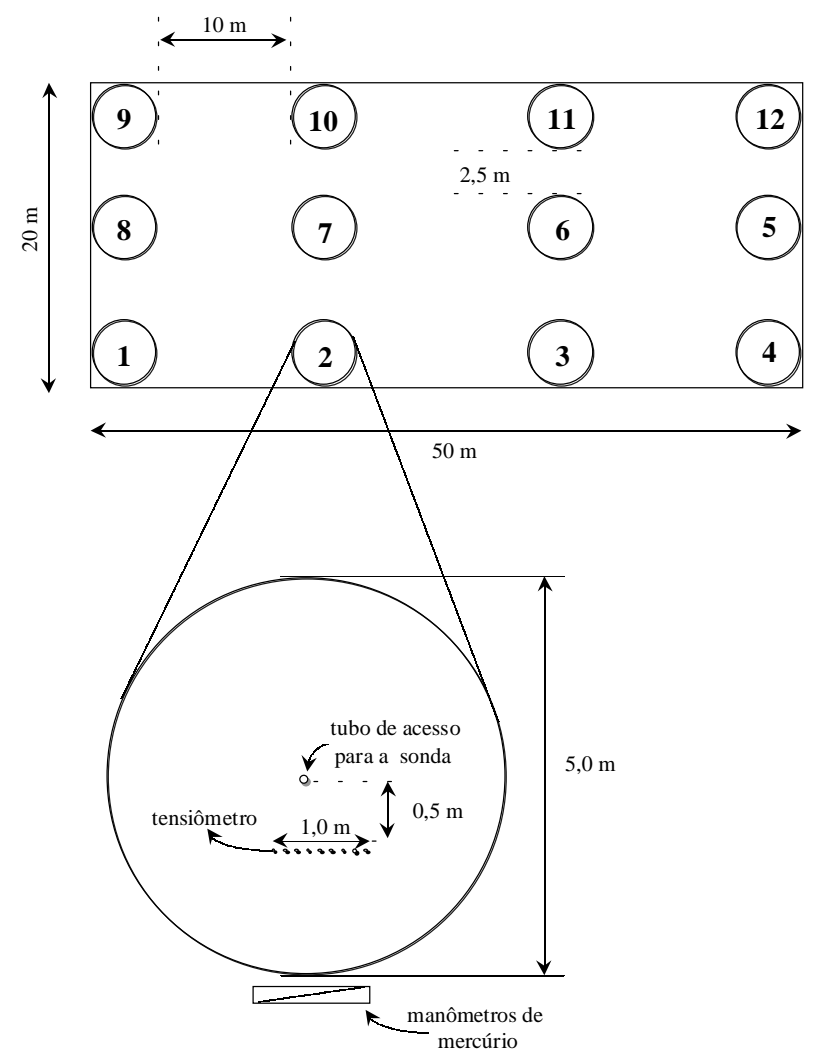

Figura 1. Esquema da área experimental mostrando a localização relativa dos doze locais de observação e, em detalhe, a configuração de um local de observação. termos de uma contagem relativa à contagem dentro da blindagem da própria sonda, determinada após cada série de observações, resultando em valores típicos em torno de 1,35.104.

Para determinar a relação entre a contagem relativa (CR) e a umidade do solo, utilizaram-se as equações lineares determinadas por Moraes et al. (1998), obtidas para cada profundidade por meio de amostragens simultâneas com a leitura da sonda em al gumas datas ao longo da execução do experimento, em diferentes pontos de medição.

Instalados os tensiômetros etubos de acesso, uma chapa de ferro galvanizado foi cravada no solo em volta decada ponto de observação atéà profundidade de aproximadamente $0,20 \mathrm{~m}$, permitindo a manutenção de uma lâmina de água no local, de tal modo que osolo nolocal setornassetãoúmi do quanto possível. Atingida a condição quase saturada, verificada pela leitura dos tensiômetros, a infiltração foi interrompida sendo a superfície do solo coberta com duas camadas de lona plástica, satisfazendo, assim, a primeira condição de contorno. A partir desse momento $(t=0)$, a umidade do solo e o potencial mátrico, a cada $0,1 \mathrm{~m}$ de profundidade, foram monitorados por mei o de leituras com a sonda de nêutrons e dos tensiômetros. O intervalo entre duas leituras variou de uma hora, no início do experimento, a dois dias, no fim do experimento, quando o processo já havia se tornado muito lento, ocorrendo, aproximadamente, 50 dias ou $1.200 \mathrm{~h}$ após o início do experimento.

Assim, para cada ponto de observação, obteve-se um conjunto de potenciais mátricos e umidades em nove profundidades, para uma série de tempos.

\section{Análise computacional}

As leituras de cada um dos 12 locais foram analisadas, utilizando um programa de computador desenvolvido no âmbito deste trabalho, cujo procedimento é apresentado a seguir:

Quadro 1. Caracterização pedológica do solo da área experimental, no campus "Luiz de Queiroz", classificado como Terra Roxa Estruturada latossólica

\begin{tabular}{|c|c|c|c|c|c|}
\hline \multirow{2}{*}{ Característica analisada } & \multicolumn{5}{|c|}{ Horizonte } \\
\hline & Ap & $\mathbf{B t}_{1}$ & $\mathbf{B t}_{2}$ & $B \mathbf{w}_{1}$ & $\mathbf{B} \mathbf{w}_{2}$ \\
\hline Profundidade (m) & $0-0,20$ & $0,20-0,76$ & $0,76-1,13$ & $1,13-1,30$ & $1,30-1,65+$ \\
\hline Textura & argilosa & argilosa & argilosa & argilosa & argilosa \\
\hline Estrutura & deformada & $\begin{array}{c}\text { blocos } \\
\text { subarredondados } \\
\text { pequenos }\end{array}$ & $\begin{array}{c}\text { blocos } \\
\text { subarredondados } \\
\text { médios }\end{array}$ & $\begin{array}{c}\text { blocos } \\
\text { subangulares } \\
\text { médios }\end{array}$ & $\begin{array}{c}\text { blocos } \\
\text { subangulares } \\
\text { médios }\end{array}$ \\
\hline Cerosidade & ausente & forte & moderada & ausente & ausente \\
\hline Densidade do solo $\left(\mathrm{kg} \mathrm{m}^{-3}\right)$ & 1.560 & 1.380 & 1.230 & 1.210 & 1.230 \\
\hline
\end{tabular}


Os valores de $\theta$ em função do tempo (t, h) são ajustados, para cada profundidade, à equação

$$
\theta=\mathrm{n}_{\mathrm{z}} \mathrm{t}^{\mathrm{z}}
$$

em que $m_{z}$ e $n_{z}$ são os coeficientes de ajuste da equação. $m_{z}$ é adiversional, ea unidade de $n_{z}$ é ${ }^{-m_{z}}$ ). Em função da equação 5, tem-se que

$$
\left.\frac{\partial \theta}{\partial \mathrm{t}}\right|_{\mathrm{z}}=\mathrm{n}_{\mathrm{z}} \mathrm{m}_{\mathrm{z}} \mathrm{t}^{\mathrm{m}_{\mathrm{z}}-1}
$$

e, com base nessa equação, estima-se o termo $\partial \mathrm{h}_{z} / \partial \mathrm{t}$ da equação 3, pela expressão

$$
\frac{\partial \mathrm{h}_{\mathrm{z}}}{\partial \mathrm{t}}=\left.\sum_{\mathrm{i}=1}^{\mathrm{z}} \mathrm{z}_{\mathrm{i}} \frac{\partial \theta}{\partial \mathrm{t}}\right|_{\mathrm{i}}=\sum_{\mathrm{i}=1}^{\mathrm{z}} \mathrm{z}_{\mathrm{i}} \mathrm{n}_{\mathrm{i}} \mathrm{m}_{\mathrm{i}} \mathrm{m}^{\mathrm{i}}-1
$$

em que $z_{i}(m)$ éa espessura da camada i. A partir da soma dos valores dos potenciais mátrico e gravitacional obtém-se o potencial total da água no solo $\left(\psi_{\mathrm{t}}, \mathrm{kPa}\right)$ em cada tempo e profundidade, o qual é ajustado, para cada tempo, à equação

$$
\psi_{\mathrm{t}}=\mathrm{p}_{\mathrm{t}}+\mathrm{q}_{\mathrm{t}} \mathrm{z}+\mathrm{r}_{\mathrm{t}} \mathrm{z}^{2}
$$

em que $p_{t}(k P a), q_{t}\left(k P a m^{-1}\right)$ e $r_{t}\left(k P a m^{-2}\right)$ são os coeficientes de ajuste da equação. Em função da equação 8 , tem-se que

$$
\left.\frac{\partial \psi_{\mathrm{t}}}{\partial \mathrm{z}}\right|_{\mathrm{t}}=\mathrm{q}_{\mathrm{t}}+2 \mathrm{r}_{\mathrm{t}} \mathrm{z}
$$

Por meio das equações 7 e9, calculam-se, para cada t ez, os dois el ementos do membro direito da equação 3 , obtendo o val or de K $(\theta)$ para a umidade correspondente. A partir do conjunto de pares de dados $(K, \theta)$ assim obtidos, estimam-se os parâmetros $K_{0}$ e $\gamma$ da equação 4 por meio de regressãolinear, tomando como variável dependente o In[K $(\theta)]$ e como variável independente o $\theta-\theta_{0}$, pois a equação 4 equivale a

$$
\ln [K(\theta)]=\ln \left[\mathrm{K}_{0}\right]+\gamma\left(\theta-\theta_{0}\right)
$$

\section{RESULTADOS E DISCUSSÃO}

\section{Análise computacional}

Os dados obtidos ajustaram-se muito bem às equações 5 e8, com val ores de R 2 mai ores do que 0,9 para todos os casos da equação 5 e para $93 \%$ dos casos da equação 8. N as figuras 2 e 3 , são mostrados exemplos dos pontos observados e linhas ajustadas para essas equações. Em conseqüência dos altos valores de $\mathrm{R}^{2}$, tem-se bastante segurança da confiabilidade dos valores de $\partial \theta / \partial t$ e $\partial \Psi t / \partial z$, calculados com base nessas equações.

$\mathrm{O}$ ajuste dos valores de $\mathrm{K}$ e $\theta$ à equação 10 , para as nove profundidades avaliadas, também resultou em valores de R2 muito altos (Quadro 2). Apenas para as primeiras duas profundidades avaliadas $(0,2$ e 0,3 m), os coeficientes de ajuste médio foram menores que 0,99. Na profundidade de 0,2 $\mathrm{m}$, 0 menor valor observado foi de 0,454 e, na de 0,3 m, o menor valor foi de 0,775 . Na figura 4, é mostrado um exemplo de um ajuste a essa equação. Os val ores dos parâmetros $\gamma$ e $K_{0}$, resultantes desses ajustes, mostraram-se, porém, muito variáveis (Quadro 3), confirmando tendência de alta variabilidade, como encontrada por Vieira etal. (1981) eBouma et al. (1989). Por exemplo, numa mesma profundidade de 0,3 m, ocorreram valores para $\mathrm{K}_{0}$ da ordem de grandeza de $10^{-8} \mathrm{~m}^{2} \mathrm{~h}^{-1} \mathrm{kPa}^{-1}$ (local 10) a $10^{2} \mathrm{~m}^{2} \mathrm{~h}^{-1} \mathrm{kPa}^{-1}$ (local 4). Para o $\gamma$, a variação foi da ordem de 200 a 6.000 , para a mesma profundidade. Tal fato, revelado para as demais profundidades, mostrou variabilidade

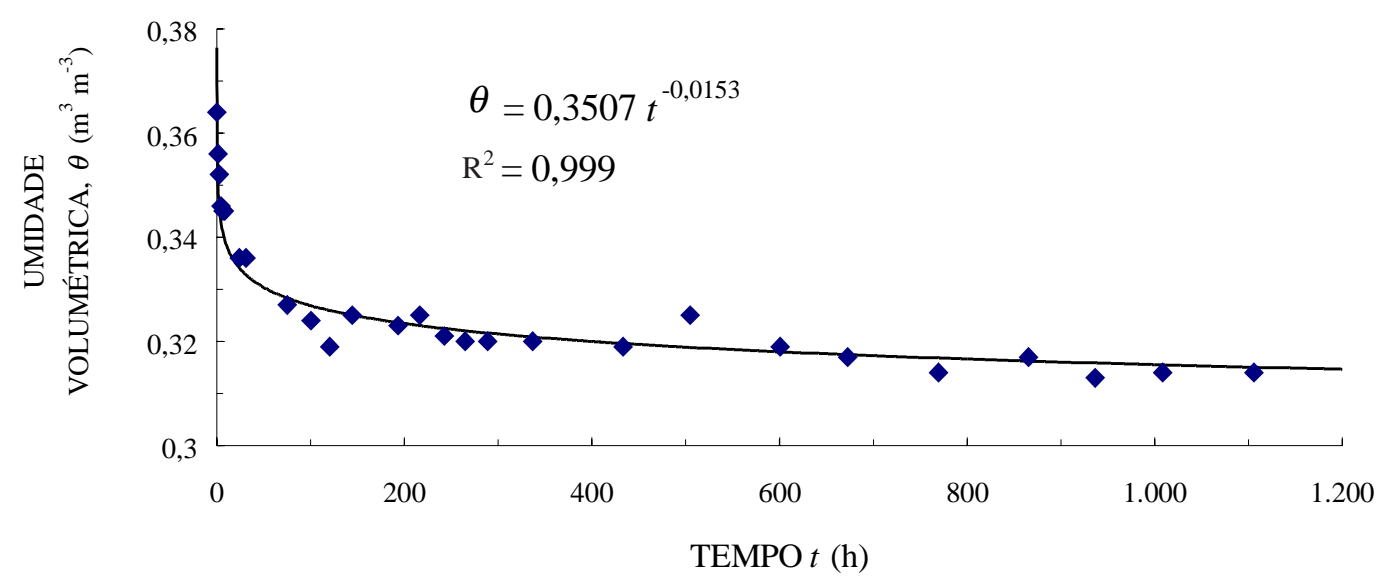

Figura 2. Ajuste à equação 5 dos dados do local 9 e profundidade de 0,8 m. 


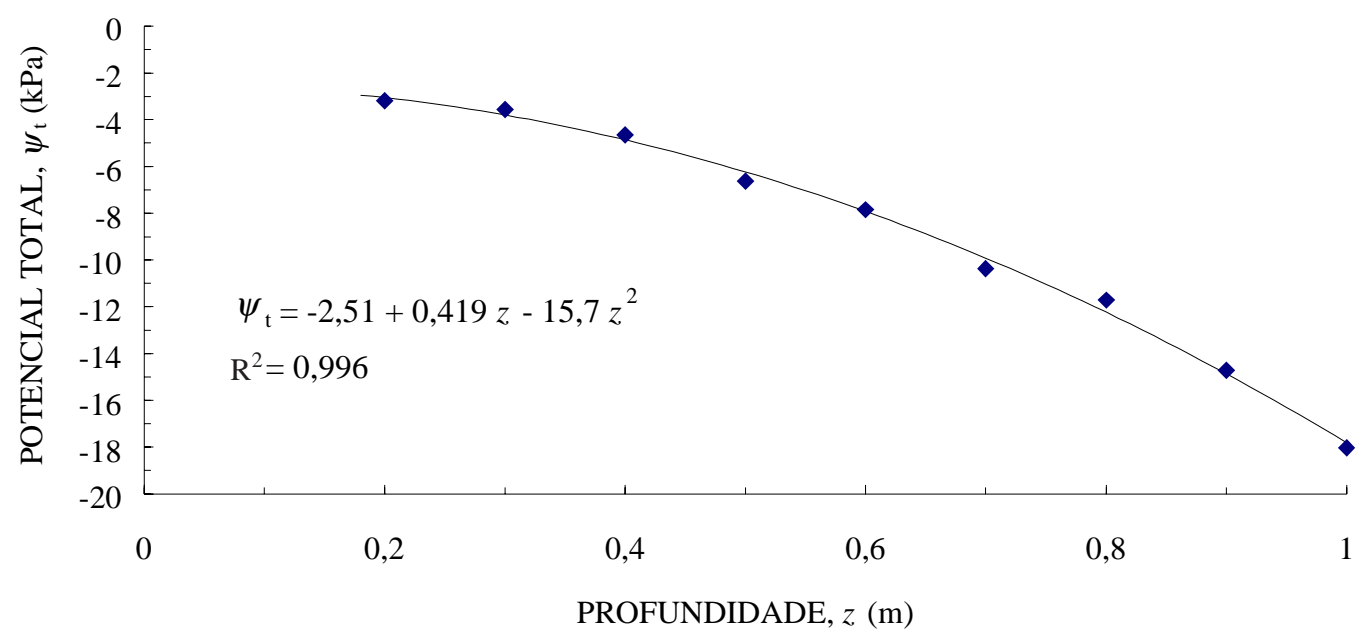

Figura 3. Ajuste à equação 8 dos dados do local 9 e tempo de 110,3 h.

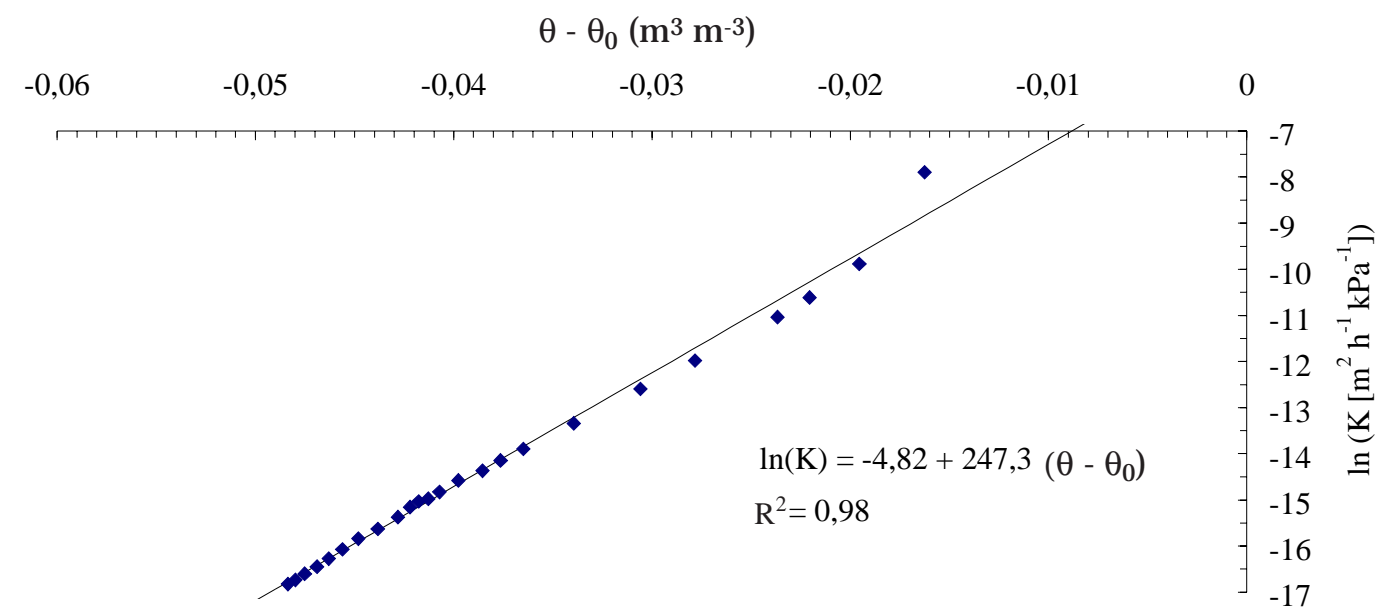

Figura 4. Ajuste à equação 10 dos dados do local 9 e profundidade de $0,8 \mathrm{~m}$.

Quadro 2. Coeficientes de determinação do ajuste dos valores de $K(\theta)$ e $\theta$ à equação 10 , para as nove profundidades e doze locais avaliados

\section{Profundidade Coeficiente de determinação médio}

\begin{tabular}{ll}
\hline $\mathrm{m}$ & \\
0,2 & 0,786 \\
0,3 & 0,965 \\
0,4 & 0,997 \\
0,5 & 0,999 \\
0,6 & 0,999 \\
0,7 & 0,997 \\
0,8 & 0,998 \\
0,9 & 0,996 \\
1,0 & 0,997 \\
\hline
\end{tabular}

muito alta da propriedade em estudo, mesmo em distâncias pequenas. Coeficientes de variação foram da ordem degrandeza de 1 para e de 3 para $\mathrm{K}_{0}$, ou seja, odesvio-padrãodesses parâmetros foi uma etrês vezes, respectivamente, maior que a sua média (Quadro 4).

Observando os dados do quadro 3, apesar de o experimento ter durado quase dois meses, em várias profundidades, notadamentenas de 0,5, 0,6 e 0,7 m, quase não ocorreu redução da umidade do solo. Tal fato põe em questão a confiabilidade da aplicação do método nestes casos, como também pode ter afetado a calibração da sonda de nêutrons. Com o objetivo de eliminar valores menos confiáveis para a análise dos resultados, admitiu-se, como critério, que a faixa de umidade $\left(\Delta \theta, \mathrm{m}^{3} \mathrm{~m}^{-3}\right)$ disponível para a determinação deveria ter sido maior que 0,03 . 
Quadro 3. Valores dos parâmetros $\gamma_{1} K_{0}\left(\mathrm{~m}^{2} \mathrm{~h}^{-1} \mathrm{kPa}^{-1}\right)$ e $\theta_{0}\left(\mathrm{~m}^{3} \mathrm{~m}^{-3}\right)$ das equações 4 e 10, para as nove profundidades e doze locais avaliados, e a variação da umidade ao longo do experimento $\left(\Delta \theta, \mathrm{m}^{3} \mathrm{~m}^{-3}\right)$ disponível para a determinação. Observações com fundo cinza referem-se aos locais em que $\Delta \theta$ foi inferior ou igual a $0,03 \mathrm{~m}^{3} \mathrm{~m}^{-3}$

\begin{tabular}{|c|c|c|c|c|c|c|c|c|c|c|}
\hline \multirow{2}{*}{ Local } & & \multicolumn{9}{|c|}{ Profundidade $(\mathrm{m})$} \\
\hline & & 0,2 & 0,3 & 0,4 & 0,5 & 0,6 & 0,7 & 0,8 & 0,9 & 1,0 \\
\hline \multirow[t]{4}{*}{1} & $\gamma$ & 149 & 262 & 828 & 853 & 644 & 549 & 545 & 396 & 213 \\
\hline & $K_{0}$ & $8,5810^{-3}$ & $5,1010^{-2}$ & $9,6710^{-1}$ & $1,2010^{-1}$ & $1,5910^{-2}$ & $9,2510^{-2}$ & $4,7510^{-2}$ & $1,9310^{-2}$ & $1,0410^{-1}$ \\
\hline & $\theta_{0}^{0}$ & 0,427 & 0,388 & 0,377 & 0,383 & 0,388 & 0,406 & 0,363 & 0,356 & 0,383 \\
\hline & $\Delta \theta$ & 0,13 & 0,07 & 0,02 & 0,02 & 0,02 & 0,03 & 0,03 & 0,04 & 0,07 \\
\hline \multirow[t]{4}{*}{2} & $\gamma$ & 196 & 385 & 490 & 1042 & 1563 & 921 & 668 & 537 & 420 \\
\hline & $K_{0}$ & $4,5110^{-4}$ & $2,7910^{-3}$ & $2,3110^{-3}$ & $7,0010^{-3}$ & $1,4110^{-3}$ & $2,2010^{-3}$ & $2,1510^{-3}$ & $7,4210^{-3}$ & $7,5610^{-3}$ \\
\hline & $\theta_{0}^{0}$ & 0,410 & 0,382 & 0,397 & 0,412 & 0,415 & 0,426 & 0,382 & 0,384 & 0,386 \\
\hline & $\Delta^{\theta}$ & 0,10 & 0,09 & 0,04 & 0,01 & 0,01 & 0,02 & 0,02 & 0,03 & 0,03 \\
\hline \multirow[t]{4}{*}{3} & $\gamma$ & 239 & 1045 & 848 & 789 & 1057 & 480 & 291 & 259 & 232 \\
\hline & $K_{0}$ & $6,7210^{-1}$ & $5,1510^{1}$ & $7,1010^{-2}$ & $2,2010^{-1}$ & $3,1910^{-1}$ & $6,6010^{-1}$ & $7,8110^{-1}$ & $7,2510^{-1}$ & $9,3210^{-1}$ \\
\hline & $\theta_{0}^{0}$ & 0,444 & 0,377 & 0,393 & 0,409 & 0,412 & 0,435 & 0,403 & 0,411 & 0,413 \\
\hline & $\Delta^{0} \theta$ & 0,13 & 0,06 & 0,03 & 0,01 & 0,03 & 0,03 & 0,05 & 0,06 & 0,07 \\
\hline \multirow[t]{4}{*}{4} & $\gamma$ & 335 & 360 & 305 & 445 & 340 & 184 & 169 & 154 & 130 \\
\hline & $K_{0}$ & $1,2410^{4}$ & $6,7610^{2}$ & $5,7410^{1}$ & $5,6710^{-1}$ & $9,2510^{-2}$ & $7,4510^{-2}$ & $4,2110^{-2}$ & $4,7310^{-2}$ & $5,0410^{-2}$ \\
\hline & $\theta_{0}^{0}$ & 0,458 & 0,434 & 0,456 & 0,433 & 0,438 & 0,470 & 0,420 & 0,417 & 0,423 \\
\hline & $\Delta_{\Delta}^{0}$ & 0,14 & 0,10 & 0,08 & 0,04 & 0,06 & 0,08 & 0,08 & 0,08 & 0,10 \\
\hline \multirow[t]{4}{*}{5} & $\gamma$ & 196 & 786 & 1157 & 2348 & 1738 & 690 & 418 & 311 & 258 \\
\hline & $K_{0}$ & $5,0010^{-3}$ & 4,61 & $1,0110^{1}$ & $1,8810^{-2}$ & $5,1310^{-2}$ & $1,0710^{-2}$ & $1,3410^{-3}$ & $5,1010^{-3}$ & $3,7710^{-2}$ \\
\hline & $\theta_{0}^{0}$ & 0,436 & 0,408 & 0,430 & 0,418 & 0,419 & 0,430 & 0,382 & 0,390 & 0,395 \\
\hline & $\Delta^{\theta}$ & 0,10 & 0,05 & 0,03 & 0,02 & 0,02 & 0,03 & 0,04 & 0,05 & 0,05 \\
\hline \multirow[t]{4}{*}{6} & $\gamma$ & 225 & 451 & 495 & 813 & 922 & 536 & 428 & 276 & 203 \\
\hline & $K_{0}$ & $6,7910^{-2}$ & $4,4910^{-2}$ & $2,9910^{-2}$ & $2,2210^{-2}$ & $8,9810^{-4}$ & $6,9810^{-4}$ & $1,8910^{-3}$ & $3,7010^{-3}$ & $4,9710^{-3}$ \\
\hline & $\theta_{0}^{0}$ & 0,425 & 0,374 & 0,403 & 0,412 & 0,408 & 0,422 & 0,378 & 0,385 & 0,385 \\
\hline & $\Delta^{\theta}$ & 0,13 & 0,08 & 0,05 & 0,02 & 0,02 & 0,03 & 0,03 & 0,04 & 0,06 \\
\hline \multirow[t]{4}{*}{7} & $\gamma$ & 118 & 487 & 543 & 907 & 775 & 537 & 409 & 299 & 240 \\
\hline & $K_{0}$ & $4,6910^{-3}$ & $4,1510^{-3}$ & $1,4410^{-2}$ & $3,1910^{-2}$ & $8,5810^{-3}$ & $2,7210^{-3}$ & $1,0310^{-2}$ & $1,0510^{-2}$ & $1,4010^{-2}$ \\
\hline & $\theta_{0}^{0}$ & 0,439 & 0,388 & 0,400 & 0,400 & 0,401 & 0,406 & 0,363 & 0,372 & 0,357 \\
\hline & $\Delta_{\Delta}^{0}$ & 0,11 & 0,05 & 0,04 & 0,02 & 0,02 & 0,02 & 0,03 & 0,05 & 0,05 \\
\hline \multirow[t]{4}{*}{8} & $\gamma$ & 124 & 524 & 1002 & 1055 & 1133 & 554 & 380 & 309 & 268 \\
\hline & $K_{0}$ & 1,03 & 1,38 & $3,0310^{-3}$ & $6,8410^{-3}$ & $1,41 \quad 10^{-3}$ & $7,3810^{-4}$ & $1,5210^{-3}$ & $1,3210^{-3}$ & $3,2810^{-3}$ \\
\hline & $\theta_{0}^{0}$ & 0,479 & 0,401 & 0,407 & 0,413 & 0,416 & 0,429 & 0,385 & 0,387 & 0,390 \\
\hline & $\Delta^{\theta}$ & 0,21 & 0,09 & 0,03 & 0,02 & 0,02 & 0,02 & 0,03 & 0,03 & 0,05 \\
\hline \multirow[t]{4}{*}{9} & $\gamma$ & 442 & 960 & 674 & 732 & 526 & 321 & 247 & 207 & 181 \\
\hline & $K_{0}$ & $2,8710^{-4}$ & $2,6710^{-5}$ & $1,7310^{-3}$ & $9,3010^{-5}$ & $1,1810^{-3}$ & $3,3510^{-3}$ & $7,7110^{-3}$ & $1,2810^{-2}$ & $7,3610^{-3}$ \\
\hline & $\theta^{0}$ & 0,438 & 0,392 & 0,403 & 0,397 & 0,398 & 0,403 & 0,364 & 0,380 & 0,386 \\
\hline & $\Delta^{0} \theta$ & 0,06 & 0,02 & 0,02 & 0,02 & 0,03 & 0,04 & 0,05 & 0,06 & 0,07 \\
\hline \multirow[t]{4}{*}{10} & $\gamma$ & 276 & 6128 & 3691 & 1888 & 920 & 332 & 316 & 235 & 216 \\
\hline & $K_{0}$ & $1,0010^{-2}$ & $2,6210^{-8}$ & $3,2410^{-8}$ & $7,0310^{-9}$ & $7,8810^{-5}$ & $5,6310^{-4}$ & $5,1710^{-4}$ & $2,3010^{-3}$ & $2,7510^{-3}$ \\
\hline & $\theta_{0}^{0}$ & 0,449 & 0,406 & 0,410 & 0,402 & 0,405 & 0,428 & 0,376 & 0,389 & 0,387 \\
\hline & $\Delta^{0} \theta$ & 0,11 & 0,03 & 0,02 & 0,01 & 0,02 & 0,03 & 0,06 & 0,05 & 0,05 \\
\hline \multirow[t]{4}{*}{11} & $\gamma$ & 49 & 574 & 5973 & -3855 & 1596 & 536 & 332 & 210 & 159 \\
\hline & $K_{0}$ & $4,1410^{-4}$ & 1,84 & 5,50 & $8,4710^{-2}$ & $2,5610^{-2}$ & $4,6710^{-3}$ & $5,11 \quad 10^{-3}$ & $1,3010^{-2}$ & $1,8410^{-2}$ \\
\hline & $\theta^{0}$ & 0,434 & 0,394 & 0,418 & 0,406 & 0,409 & 0,419 & 0,372 & 0,381 & 0,401 \\
\hline & $\Delta^{\theta}$ & 0,15 & 0,06 & 0,03 & 0,01 & 0,01 & 0,03 & 0,06 & 0,07 & 0,08 \\
\hline \multirow[t]{4}{*}{12} & $\gamma$ & 168 & 248 & 405 & 764 & 560 & 329 & 322 & 265 & 221 \\
\hline & $K_{0}$ & 3,99 & $1,0010^{-1}$ & 1,14 & $1,6010^{-1}$ & $1,5210^{-2}$ & $4,9410^{-3}$ & $2,9710^{-3}$ & $4,2510^{-3}$ & $3,0210^{-3}$ \\
\hline & $\theta^{0}$ & 0,461 & 0,408 & 0,432 & 0,421 & 0,422 & 0,437 & 0,385 & 0,389 & 0,392 \\
\hline & $\Delta^{0} \theta$ & 0,19 & 0,08 & 0,05 & 0,02 & 0,04 & 0,04 & 0,06 & 0,09 & 0,05 \\
\hline
\end{tabular}


Quadro 4. Médias e coeficientes de variação (cv) dos parâmetros $\gamma, K_{0}\left(m^{2} h^{-1} k P a-1\right), \theta_{0}\left(m^{3} m^{-3}\right)$ das equações 4 e 10; coeficientes de variação (C.V.) dos valores de $K_{0}$ corrigidos para a menor umidade inicial observada na profundidade $\left(K_{0}\right.$-corr., $\left.\mathrm{m}^{2} \mathrm{~h}^{-1} \mathrm{kPa}^{-1}\right)$; coeficientes de correlação $(r)$ da relação entre $\theta_{0}$ e $K_{0}$, para as nove profundidades e doze locais avaliados, para todos os locais e para aqueles em que a variação da umidade ao longo do experimento $(\Delta \theta)$ foi superior a $0,03 \mathrm{~m}^{3} \mathrm{~m}^{-3}$

\begin{tabular}{|c|c|c|c|c|c|c|c|c|c|c|}
\hline \multirow{2}{*}{ Local } & & \multicolumn{9}{|c|}{ Profundidade (m) } \\
\hline & & 0,2 & 0,3 & 0,4 & 0,5 & 0,6 & 0,7 & 0,8 & 0,9 & 1,0 \\
\hline \multirow{3}{*}{$\begin{array}{l}\text { M édia } \\
\text { (Todos) }\end{array}$} & $\gamma$ & 210 & 1017 & 1368 & 648 & 981 & 497 & 377 & 288 & 228 \\
\hline & $\mathrm{K}_{0}$ & $1,0310^{3}$ & $6,1310^{1}$ & 6,27 & $1,0310^{-1}$ & $4,4410^{-2}$ & $7,1510^{-2}$ & $7,5310^{-2}$ & $7,1010^{-2}$ & $9,8810^{-2}$ \\
\hline & $\theta_{0}$ & 0,442 & 0,396 & 0,410 & 0,409 & 0,411 & 0,426 & 0,381 & 0,387 & 0,392 \\
\hline \multirow{3}{*}{$\begin{array}{c}\text { C.V. } \\
\text { (Todos) }\end{array}$} & $\gamma$ & 0,50 & 1,60 & 1,25 & 2,34 & 0,46 & 0,39 & 0,35 & 0,35 & 0,32 \\
\hline & $\mathrm{K}_{0}$ & 3,46 & 3,17 & 2,62 & 1,58 & 2,04 & 2,63 & 2,96 & 2,91 & 2,67 \\
\hline & $\theta_{0}$ & 0,041 & 0,042 & 0,051 & 0,031 & 0,031 & 0,042 & 0,044 & 0,041 & 0,042 \\
\hline \multirow{3}{*}{$\begin{array}{c}\text { M édia } \\
(\Delta \theta>0,03)\end{array}$} & $\gamma$ & 210 & 512 & 447 & 445 & 450 & 278 & 299 & 261 & 211 \\
\hline & $\mathrm{K}_{0}$ & $1,0310^{3}$ & $7,3610^{1}$ & $1,1710^{1}$ & $5,6710^{-1}$ & $5,3910^{-2}$ & $2,7610^{-2}$ & $1,2010^{-1}$ & $8,4310^{-2}$ & $1,0710^{-1}$ \\
\hline & $\theta_{0}$ & 0,442 & 0,395 & 0,418 & 0,433 & 0,430 & 0,437 & 0,386 & 0,387 & 0,392 \\
\hline \multirow{4}{*}{$\begin{array}{c}\text { C.V. } \\
(\Delta \theta>0,03)\end{array}$} & $\gamma$ & 0,50 & 0,48 & 0,21 & - & 0,35 & 0,29 & 0,26 & 0,26 & 0,20 \\
\hline & $\mathrm{K}_{0}$ & 3,46 & 2,89 & 2,18 & - & 1,01 & 1,47 & 2,43 & 2,67 & 2,57 \\
\hline & $\mathrm{K}_{0}-\mathrm{corr}$ & 1,30 & 3,12 & 0,99 & - & 1,35 & 1,73 & 2,52 & 3,12 & 3,19 \\
\hline & $\theta_{0}$ & 0,041 & 0,045 & 0,062 & - & 0,027 & 0,076 & 0,050 & 0,045 & 0,044 \\
\hline (Todos) & $r$ & 0,64 & 0,21 & 0,37 & 0,32 & 0,35 & 0,29 & 0,37 & 0,42 & 0,46 \\
\hline$(\Delta \theta>0,03)$ & $r$ & 0,64 & 0,58 & 0,99 & - & 1,00 & 0,91 & 0,63 & 0,46 & 0,45 \\
\hline
\end{tabular}

Calcularam-se, então, médias e coeficientes de variação, consi derando apenas os val ores obtidos com $\Delta \theta>0,03$. O quadro 4 mostra que os coeficientes de variação de eK $_{0}$, principal mente os de $\gamma$, reduziram consi deravel mentequando adotado esse procedimento, apresentando val ores em torno de 0,5 , nas camadas superiores do solo, e em torno de 0,25, nas camadas inferiores. Os valores indicam que a maior fonte de variabilidade nas relações matemáticas entre $K$ e $\theta$ está no valor da condutividade hidráulica do solo saturado $\left(\mathrm{K}_{0}\right)$. A alta variabilidade dos parâmetros indica que a utilização de apenas uma medida da condutividadehidráulica para uma área maior pode resultar em erros muito grandes quando se pretende, por exemplo, determinar a drenagem profunda ou o balanço hídrico do solo.

A dificuldade na determinação de $K_{0}$ tem sua origem, entre outros, na heterogeneidadedos valores de $\theta_{0}$ nos 12 locais. $O$ val or de $K_{0}$ num local de observação refere-se ao $\theta_{0}$ daquele local, que pode ser diferente do $\theta_{0}$ de outro local. Verificou-se uma correlação positiva entre os valores de $K_{0}$ e $\theta_{0}$ (Quadro 4), principalmente quando considerados apenas os val ores obtidos em locais com $\Delta \theta>0,03$. Com a finalidade de investigar se as variações de $\theta_{0}$ foram determinantes para a variabilidade em $\mathrm{K}_{0}$, verificou-sese, comparando os valores deK para um mesmo $\theta$, a variabilidade diminui. Para tal, calcul ouse pela equação 4, por profundidade e para todos os pontos de observação com $\Delta \theta>0,03$, o valor de $K$ para a menor $\theta_{0}$ observada naquela profundidade (Quadro 4). Alguns valores, principalmente nas camadas superficiais, mostraram-se menores do que os determinados com os $\mathrm{K}_{0}$ não corrigidos, mas os coeficientes de variação cal culados dessa forma até aumentaram, indicando não depender da fonte da variação de $K_{0}$ da variação de $\theta_{0}$.

Graficamente, as funções $K$ versus $\theta$ podem ser observadas na figura 5 . Nessa figura, ondeas linhas grossas referem-se às regressões feitas com $\Delta \theta>0,03$, verifica-se uma variação muito maior na inclinação das linhas $(\gamma)$ nas profundidades de 0,2 e 0,3 m quenas profundidades de 0,8 a 1,0 m. Essefato, também observado no quadro 4, evidencia que os problemas na determinação da função K $-\theta$ relacionados com a variabilidade dos resultados obti dos podem ser mais expressivos nas camadas mais superficiais do que nas mais profundas. Como as camadas mais superficiais são de maior interesse em estudos de balanço hídrico e da relação solo-planta, determinações da função $K$ versus $\theta$ devem sempre ser analisadas, considerando essa grande variabilidade. 

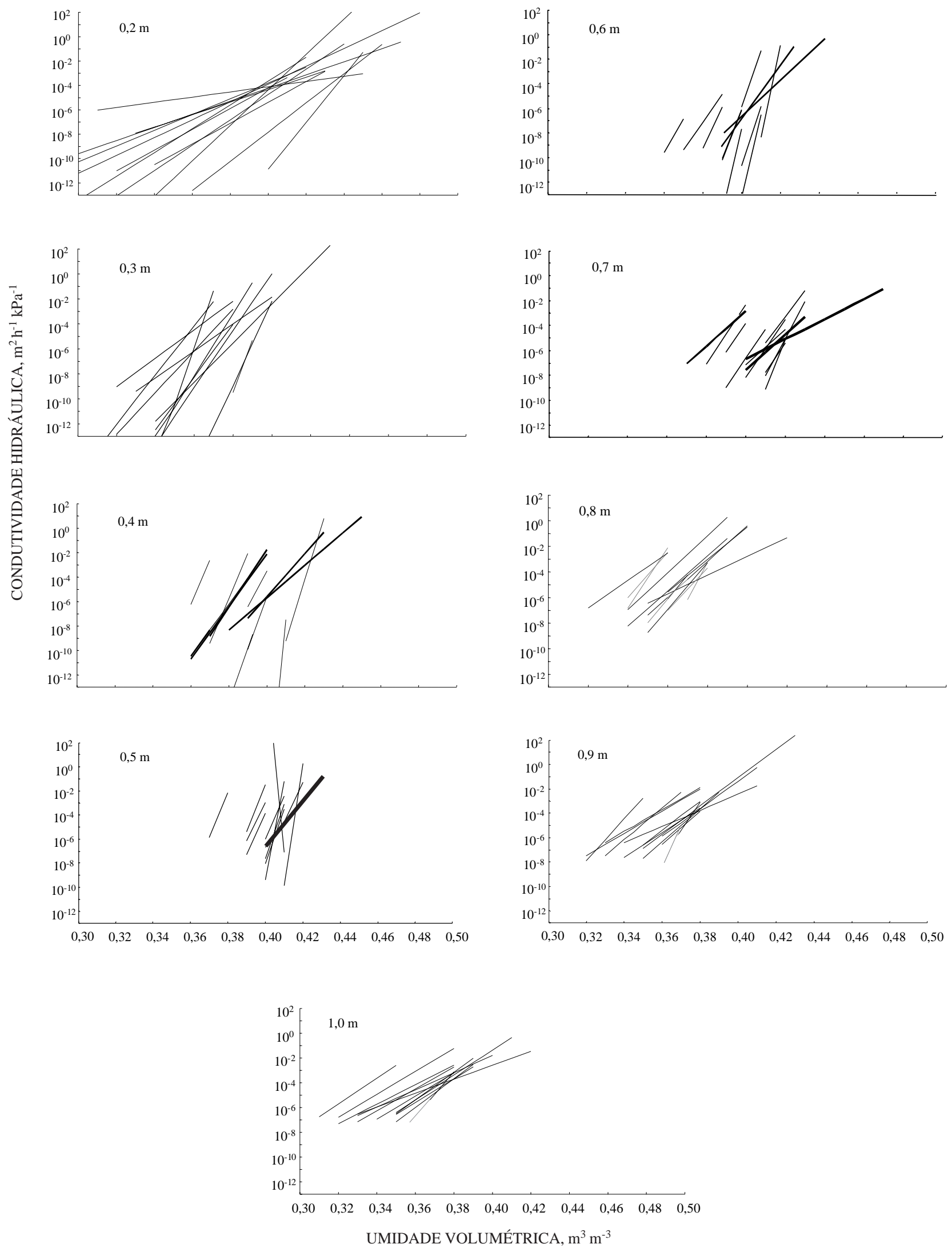

Figura 5. Condutividade hidráulica em função da umidade para os 12 locais avaliados. Linhas finas indicam observações nas quais a variação da umidade ao longo do experimento $(\Delta \theta)$ foi inferior ou igual a 0,03; linhas grossas referem-se a observações $\operatorname{com} \Delta \theta>0,03$. 


\section{CONCLUSÕES}

1. Os valores de umidade versus tempo no experimento de drenagem interna ajustam-se muito bem ( $R^{2}>0,9$ para todos os casos avaliados) a uma equação potencial.

2. No experimento de drenagem interna, os valores de potencial total versus profundidade, em determinado tempo, ajustam-se muito bem $\left(R^{2}>0,9\right.$ para mais que $90 \%$ dos casos avaliados) a uma equação polinomial de segundo grau.

3. Os valores de $K$ e $\theta$ ajustam-se muito bem à equação 4 para as camadas subsuperficiais; em alguns casos, oseu ajusteéinferior para as camadas menos profundas.

4. As grandes diferenças ocorridas entre repetições de determinações da função $K(\theta)$, pelo método do perfil instantâneo devem-se, em menor grau, a diferenças entre os val ores de $\gamma$ encontrados e, principal mente, à variabilidade muito grande dos valores de $\mathrm{K}_{0}$ estimados por esse método, conseqüência, entre outros, das diferenças nos valores de $\theta_{0}$.

5. Uma relação de $K$ versus $\theta$ determinada pelo método do perfil instantâneo, em uma área de alguns metros quadrados, não é representativa para uma área muito maior que aquela onde foi determinada, principalmente para as camadas superficiais do solo.

\section{LITERATURA CITADA}

BOUMA, J .; J ONGMANS, A. \& STEIN, A. Characterizing spatially variable hydraulic properties of a boulder clay deposit in the Netherlands. Geoderma, 45:19-29, 1989.

FREIRE, J .C. Condutividade hidráulica e capacidade de campo de Latossolo Roxo distrófico não saturado. R. Bras. Ci. Solo, 3:73-77, 1979.

GARDNER, W.R. \& EHLIG, C.F. Some observations on the movement of water to plant roots. Agron. J ., 54:453-456, 1962.

GOVINDARAJ U, R.S.; KAVVAS, M.L.; ROLSTON, D.E. \& BIGGAR, J . Error analyses of simplified unsaturated flow models under large uncertainty in hydraulic properties. Water Res. Res., 28:2913-2924, 1992.

HAINSWORTH, J.M. \& AYLMORE, L.A.G. Water extraction by single plant roots. Soil Sci. Soc. Am. J ., 50:841-848, 1986.

HAINSWORTH,J .M. \& AYLMORE, L.A.G. Non-uniform soil water extraction by plant roots. Plant Soil, 113:121-124, 1989.
HILLEL, D.A.; KRENTOS, V.K. \& STILIANOV, Y. Procedureand test of an internal drainage method for measuring soil hydraulic characteristics in situ. Soil Sci., 114:395-400, 1972.

J ONG van LIER, Q. \& LIBARDI, P.L. Extraction of soil water by plants: development and validation of a model. R. Bras. Ci. Solo, 21:535-542, 1997.

KUNZE, R.J . \& NIELSEN, D.R. Comparison of soil water infiltration profiles obtained experimentally and by solution of Richards equation. Soil Sci., 135:342-349, 1983.

LIBARDI, P.L.; REICHARDT, K.; NIELSEN, D.R. \& BIGGAR, J.W. Simplified field methods for estimating the unsaturated hydraulic conductivity. Soil Sci. Soc. Am. J ., 44:3-6, 1980.

LIBARDI, P.L. \& SAAD, A.M. Balanço hídrico em cultura defeijão irrigada por pivô central em Latossolo Roxo. R. Bras. Ci. Solo, 18:529-532, 1994.

LOGSDON, S.D. \& J AYNES, D.B. Spatial variability of hydraulic conductivity in a cultivated field at different times. Soil Sci. Soc. Am. J ., 60:703-709, 1996.

MORAES, S.O. \& LIBARDI, P.L. Variabilidadeda água disponível de uma Terra Roxa Estruturada Iatossólica. Sci. Agric., 50:404-412, 1993.

MORAES, S.O.; LIBARDI, P.L. \& MARCIANO, C.R. Seleção da curva de calibração de sonda de nêutrons mais adequada ao longo do perfil do solo. R. Bras. Ci. Solo, 1999.

REYNOLD, W.D. \& ZEBCHUK, W.D. Hydraulic conductivity in a clay soil: two measurement techniques and spatial characterization. Soil Sci. Soc. Am. J ., 60:1679-1685, 1996.

RUSSO, D.; RUSSO, I. \& LAUFER, A. On the spatial variability of parameters of the unsaturated hydraulic conductivity. Water Res. Res., 33,947-956, 1997.

SWARTZENDRUBER, D. A quasi-solution of Richards equation for the downward infiltration of water into soil. Water Res. Res., 23:809-817, 1987.

VIEIRA, S.R.; NIELSEN, D.R. \& BIGGAR, J .W. Spatial variability of field-measured infiltration rate. Soil Sci. Soc. Am. J., 45:1040-8, 1981.

WARRICK, A.W. \& NIELSEN, D.R. Spatial variability of soil physical properties in the field. In: HILLEL, D., ed. Applications of soil physics. New York, Academic Press, 1980. p.319-344.

WATSON, K.K. An instantaneous profilemethod for determining the hydraulic conductivity of unsaturated porous materials. Water Res. Res., 2:709-15, 1966

WEBSTER, R. \& OLIVER, M.A. Sample adequately to estimate variograms of soil properties. J . Soil Sci., 43:177-192, 1992.

WOLFE, M.L.; LARSON, C.L. \& ONSTAD, C.A. Hydraulic conductivity and Green-Ampt Infiltration modeling for tilled soils. Trans. Am. Sci. Agric. Eng., 31:1135-1140, 1988. 